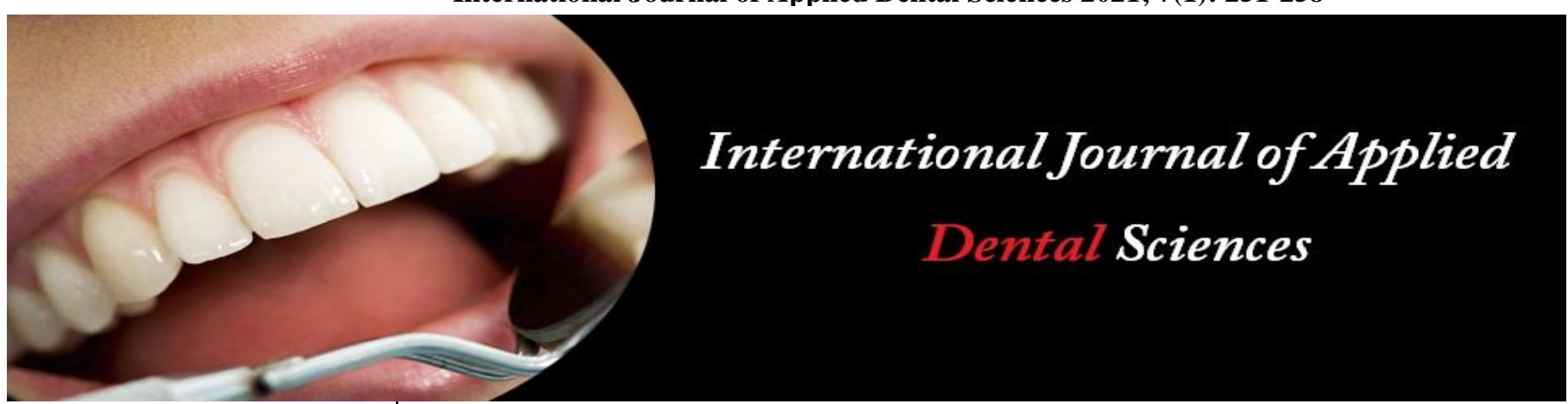

ISSN Print: 2394-7489

ISSN Online: 2394-7497

IJADS 2021; 7(1): 251-258

(C) 2021 IJADS

www.oraljournal.com

Received: 02-11-2020

Accepted: 11-12-2020

Ramy Siam

Fixed Prosthodontics

Department, Faculty of

Dentistry, Cairo University,

Cairo, Egypt

\section{Gihan Elnaggar}

Fixed Prosthodontics

Department, Faculty of

Dentistry, Cairo University,

Cairo, Egypt

Eman Hassanien

Fixed Prosthodontics

Department, Faculty of

Dentistry, Cairo University, Cairo, Egypt
Corresponding Author:

Ramy Siam

Fixed Prosthodontics

Department, Faculty of

Dentistry, Cairo University,

Cairo, Egypt

\section{Surface roughness and translucency of glazed lithium disilicate (IPS E.Max) vs. glazed and polished "zirconia-reinforced lithium silicate" (Celtra duo) (in vitro study)}

\section{Ramy Siam, Gihan Elnaggar and Eman Hassanien}

DOI: https://doi.org/10.22271/oral.2021.v7.i1d.1141

Abstract

The aim of the present study is to evaluate the effect of finishing protocol and zirconia content on surface roughness and translucency of Zirconia-Reinforced Lithium Silicate (Celtra Duo).

Methodology: Disks (10 $\mathrm{mm}$ in diameter and $2.2 \mathrm{~mm}$ thick) were fabricated for the following groups $(n=7)$ for each group: polished Celtra Duo CAD, glazed Celtra Duo CAD and IPS e.max CAD (control group). The surface roughness $(\mathrm{Ra})$ of the specimens were measured with optical profilometer and scanning electron microscope (SEM). Additionally, the translucency (TP) of the specimens were measured with spectrophotometer. The influence of material type and surface finishing protocol on surface roughness and translucency were analyzed using a multivariate analysis, 1-way ANOVA, and the Tukey's post-hoc test $(P<.05)$.

Results: the effect of finishing protocol $(\mathrm{p}=0.8204)$ and ceramic type $(\mathrm{p}=0.4448)$ on surface roughness was non-significant among the tested groups. Whereas, the effect of ceramic type on translucency was significant $(\mathrm{p}=0.0023)$, where Celtra Duo CAD ceramics demonstrated significantly higher translucency when compared to IPS e.max CAD ceramics. However, the effect of finishing protocol on translucency of Celtra duo groups was non-significant.

Conclusion: Celtra Duo CAD is more translucent than IPS e.max CAD regardless of the finishing protocol. The surface roughness of Celtra Duo CAD and IPS e.max CAD are comparable.

Keywords: IPS e.max CAD, Celtra Duo CAD, Zirconia-Reinforced Lithium Silicate, finishing protocol, zirconia, roughness, translucency

\section{Introduction}

The patients' demand for natural looking restorations that mimic tooth structure has led to the development of new all-ceramic systems. Esthetically pleasing restoration should be an exact replica of shape, size, translucency and surface texture of the natural tooth ${ }^{[1,2]}$. The constant search for all-ceramic restorative materials that combine aesthetics and strength has contributed to the development of reinforced glass-ceramics [zirconia- reinforced lithium silicate (ZLS) glass-ceramics] such as Celtra Duo by the addition of $10 \%$ zirconia crystals by weight. Pursuant to the manufacturers, the flexural strength and hardness of ZLS Celtra Duo were higher when compared to lithium disilicate glass ceramics ${ }^{[3]}$. The surface quality of restorations is considered a key factor for clinical success. A smooth surface increases the lifetime of ceramic restorations, optimizing their esthetic appearance and reducing plaque accumulation and surface pigmentation. Limited information exists about the effect of different surface finishing protocols on the surface roughness and translucency of different ceramic systems. Polishing with an adjustment kit, polishing with an adjustment kit plus diamond polishing paste and glazing are well-known surface finishing methods ${ }^{[4]}$. However, the effect of adding zirconia to lithium silicate ceramics and the effect of finishing protocols on its roughness and translucency are still under investigation. The null hypothesis of the present study were: Ceramic type (Celtra Duo CAD vs. IPS e.max CAD) and finishing protocol (polishing vs. glazing) of zirconia reinforced lithium silicate ceramics will not affect the surface roughness and translucency when compared to lithium disilicate glass ceramics. 


\section{Materials and methods}

\section{Discs samples construction}

A total 21 ceramic discs $(n=21) 10 \mathrm{~mm}$ in diameter and 2.2 $\mathrm{mm}$ in thickness were fabricated as following: Polished zirconia reinforced lithium silicate Celtra Cuo CAD discs (group A, n=7), Glazed zirconia reinforced lithium silicate Celtra Duo CAD discs (group B, n=7) and Glazed lithium disilicate IPS e.max CAD discs (group $\mathrm{C}, \mathrm{n}=7$ ). All blocks were sliced using (IsoMet 4000) low speed micro-saw Figure (1).

All disc samples were checked carefully using magnifying lens for any surface defects. Discs with surface defects were discarded and replaced by new one. All specimens were checked using a precise digital caliper (Mitutoyo 293-821) Figure (2) at different points to verify the disc thickness. Finishing and polishing to all discs in this group was performed using (EVE DIASYNT® PLUS) set according to manufacturer's instructions starting with coarse grit rubber wheel followed by medium, fine and extra- fine grit rubber wheels were used in succession in one direction for 30 seconds at $10000 \mathrm{rpm}$, to obtain highly polished surface. Consequently, (VITA diamond restoration polish) was used to enhance surface luster Figure (3), resulting in $(2 \mathrm{~mm})$ thick discs. Discs were rechecked using precise digital caliper at different points with accuracy of $( \pm 0.05 \mathrm{~mm})$ to verify their thickness.

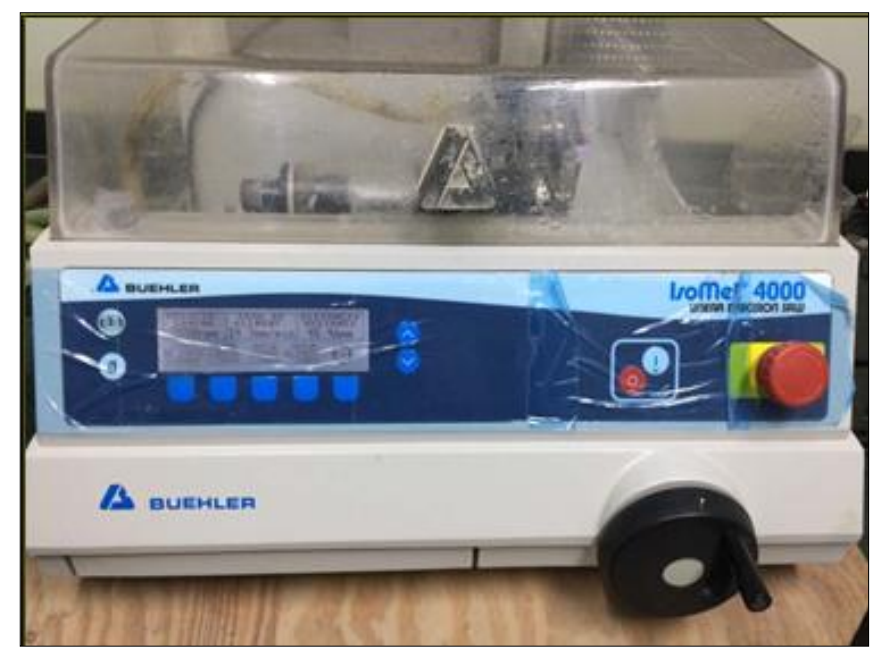

Fig 1: IsoMet 4000 linear precision saw.

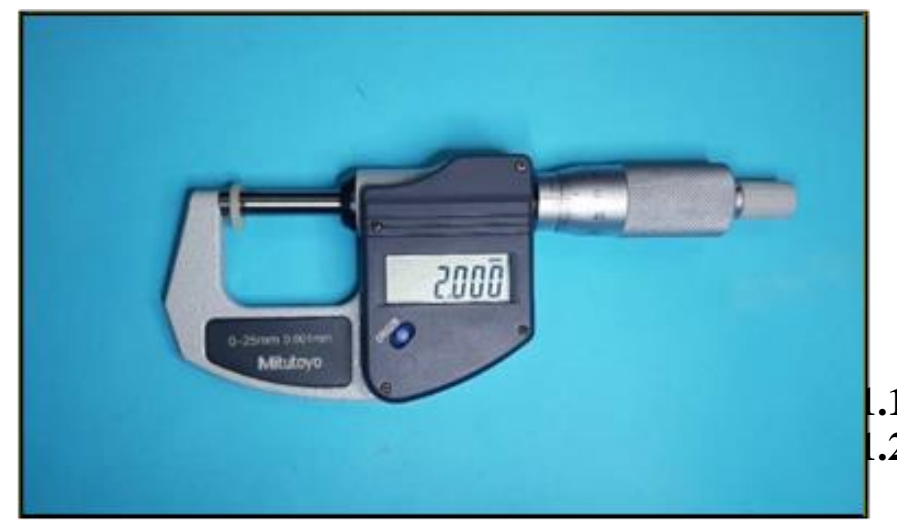

Fig 2: Measuring samples thickness after finishing and polishing.

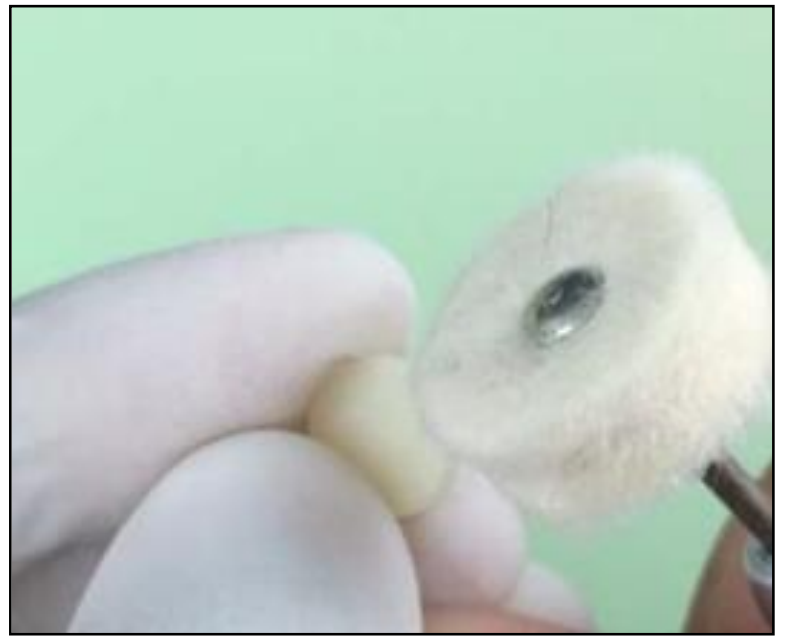

Fig 3: Polishing of discs using diamond restoration polish.

\section{Roughness testing \\ Non-contact optical profilometer}

Specimens were photographed using USB Digital microscope with a built-in camera connected with an IBM compatible personal computer using a fixed magnification of 120X. The cropped images were analyzed using (WSxM software) to calculate average of heights ( $\mathrm{Ra}$ ) expressed in $\mu \mathrm{m}$, which can be assumed as a reliable indices of surface roughness $[5,6]$. Figure (4). Within the (WSxM software), all limits, sizes, frames and measured parameters are expressed in pixels. These images aided in optical profilometer analysis. Therefore, system calibration was done to convert the pixels into absolute real-world units. Calibration was made by comparing an object of known size (a ruler in this study) with a scale generated by the software. Subsequently, a 3D image of the surface profile of the specimens was created.

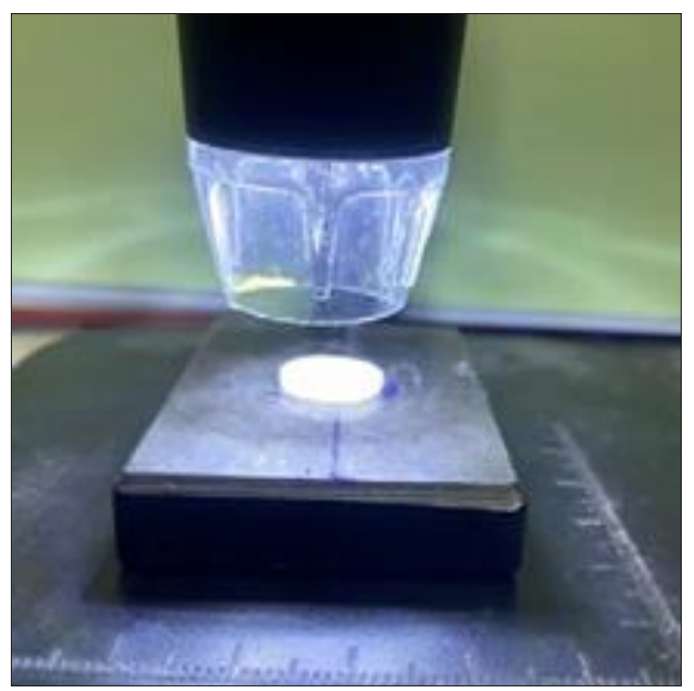

Fig 4: Disc sample in Scope Capture Digital Microscope.

\section{.1. Scanning Electron microscope (SEM)}

.2. Samples were examined using Scanning Electron Microscope (SEM) Model Quanta 250 FEG (Field Emission Gun). Figure (5) \& Figure (6) Three different magnifications (250X, 500X $\& 1000 X)$ were taken for each examined surface. 


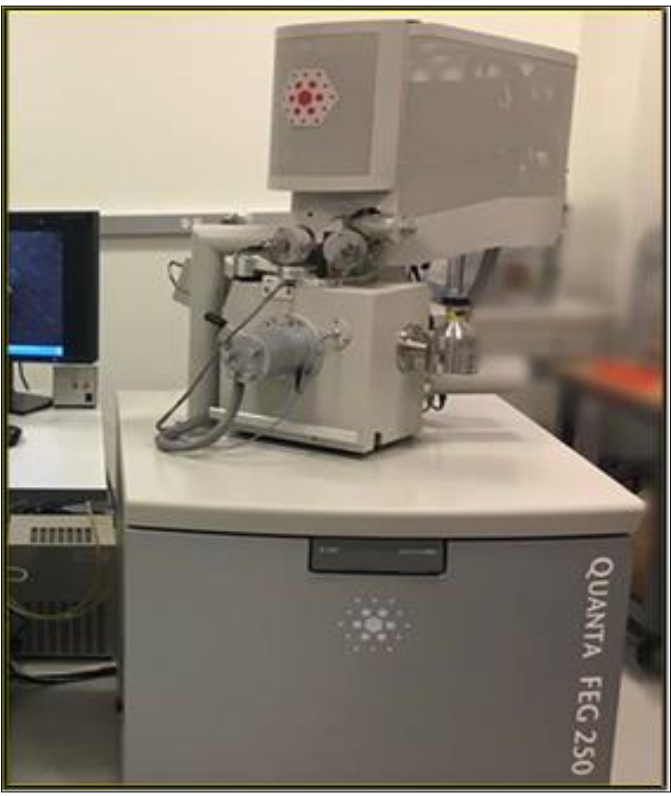

Fig 5: Quanta 250 SEM.

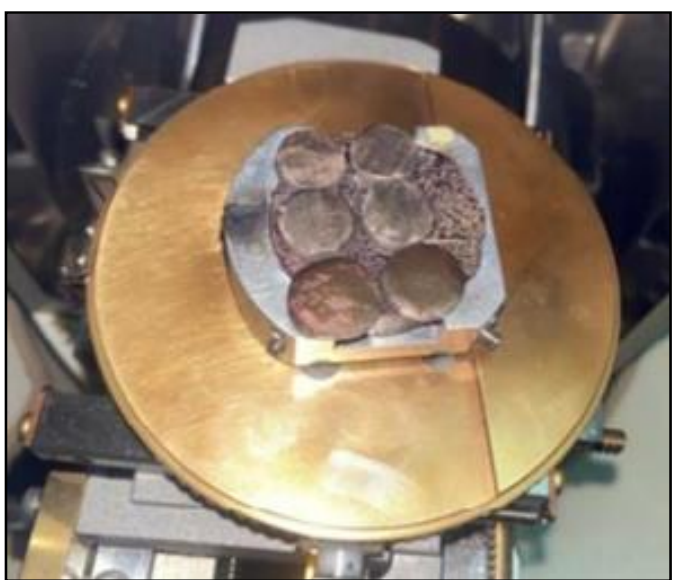

Fig 6: Disc samples in the SEM.

\section{Translucency testing}

The specimens were measured using a Reflective Spectrophotometer Figure (7). The measurements were performed at the center of each specimen over a white Teflon $\left(\mathrm{CIE} \mathrm{L} *=88.81, \mathrm{a}^{*}=-4.98, \mathrm{~b}^{*}=6.09\right)$ and black backing $(\mathrm{CIE}$ $\left.L^{*}=7.61, a^{*}=0.45, b^{*}=2.42\right)$ relative to the CIE standard illuminant (D65). The translucency parameters (TP) values were obtained by calculating the color difference of the specimens over black and white backgrounds by using the following equation: $\mathrm{TP}=\left[\left(\mathrm{Lb}^{*}-\mathrm{Lw}^{*}\right) 2+(\mathrm{ab} *-\mathrm{aw} *) 2+(\mathrm{bb} *\right.$
- bw*)2] 1/2, where letters "b" and "w" refer to color coordinates over the black and white backgrounds, respectively. The $\mathrm{L}^{*}$ values of 0 to 100 represent a black and a reference white, respectively. This coordinate is a measure of lightness-darkness of the material. The greater the $\mathrm{L}^{*}$ is, the lighter the specimen. The $a^{*}$ and $b^{*}$ values represent the redness- greenness and yellowness-blueness, respectively. Positive $a^{*}$ relates to the amount of redness and negative values relate to greenness of the specimen. $b^{*}$ coordinate is a measure of the chroma along the yellow-blue axis. Positive $b^{*}$ values relate to the amount of yellowness, while negative values relate to blueness of the specimen.

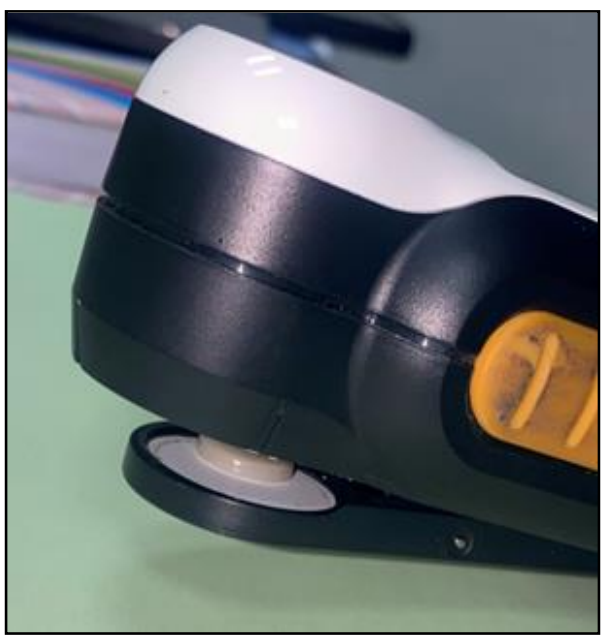

Fig 7: Disc sample in the Reflective Spectrophotometer.

\section{Results \& Discussion}

Results of the surface roughness testing using non-contact optical profilometer

Descriptive statistics showing mean values, standard deviations $( \pm \mathrm{SD})$ for averaged roughness $(\mathrm{Ra})$ measured in micron $(\mu \mathrm{m})$ recorded for the 3 tested groups are summarized in Table (1) and graphically represented in Figure (8) \& Figure (9).

It was found that Polished Celtra Duo CAD recorded the highest average roughness mean values $(0.2550 \mu \mathrm{m})$ followed by Glazed Celtra Duo CAD mean values $(0.2536 \mu \mathrm{m})$ while the lowest average roughness mean values were recorded for Glazed IPS e.max CAD $(0.2532 \mu \mathrm{m})$. The difference between groups was statistically non-significant as indicated by oneway ANOVA ( $\mathrm{p}=0.8204>0.05)$.

Table 1: Results of the surface roughness of the tested ceramics:

\begin{tabular}{|c|c|c|c|c|c|c|}
\hline \multicolumn{2}{|c|}{ Variables } & \multicolumn{2}{c|}{ Descriptive Statistics } & \multicolumn{2}{c|}{$95 \%$ Confidence Intervals } & ANOVA \\
\cline { 3 - 8 } & Mean & SD & Lower & Upper & P value \\
\hline Celtra Duo & Polished (A) & 0.2550 & 0.004 & 0.2521 & 0.258 & \\
\cline { 2 - 8 } CAD & Glazed (B) & 0.2536 & 0.005 & 0.2501 & 0.2571 & 0.8204 \\
IPS e.max
\end{tabular}




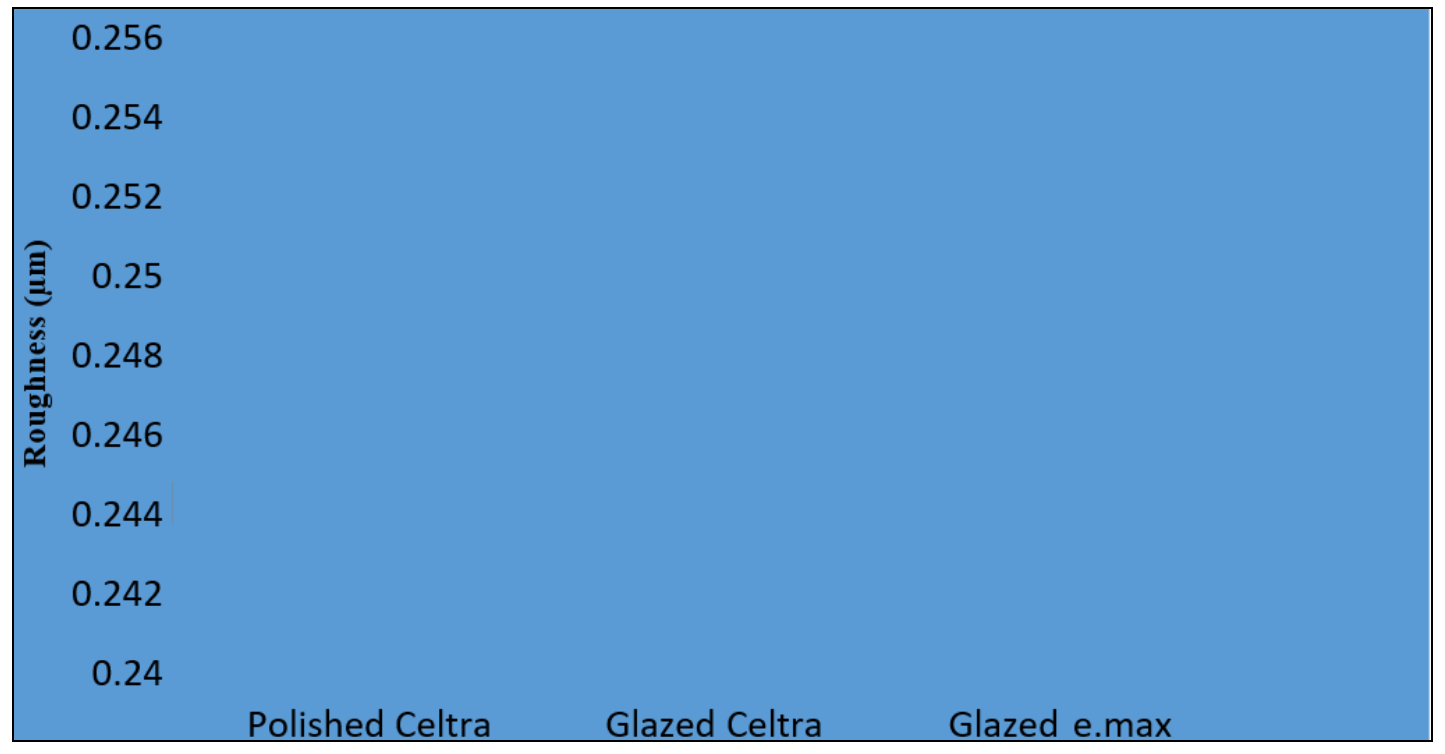

Fig 8: Column chart of the surface roughness mean values of the tested groups (A, B and C).
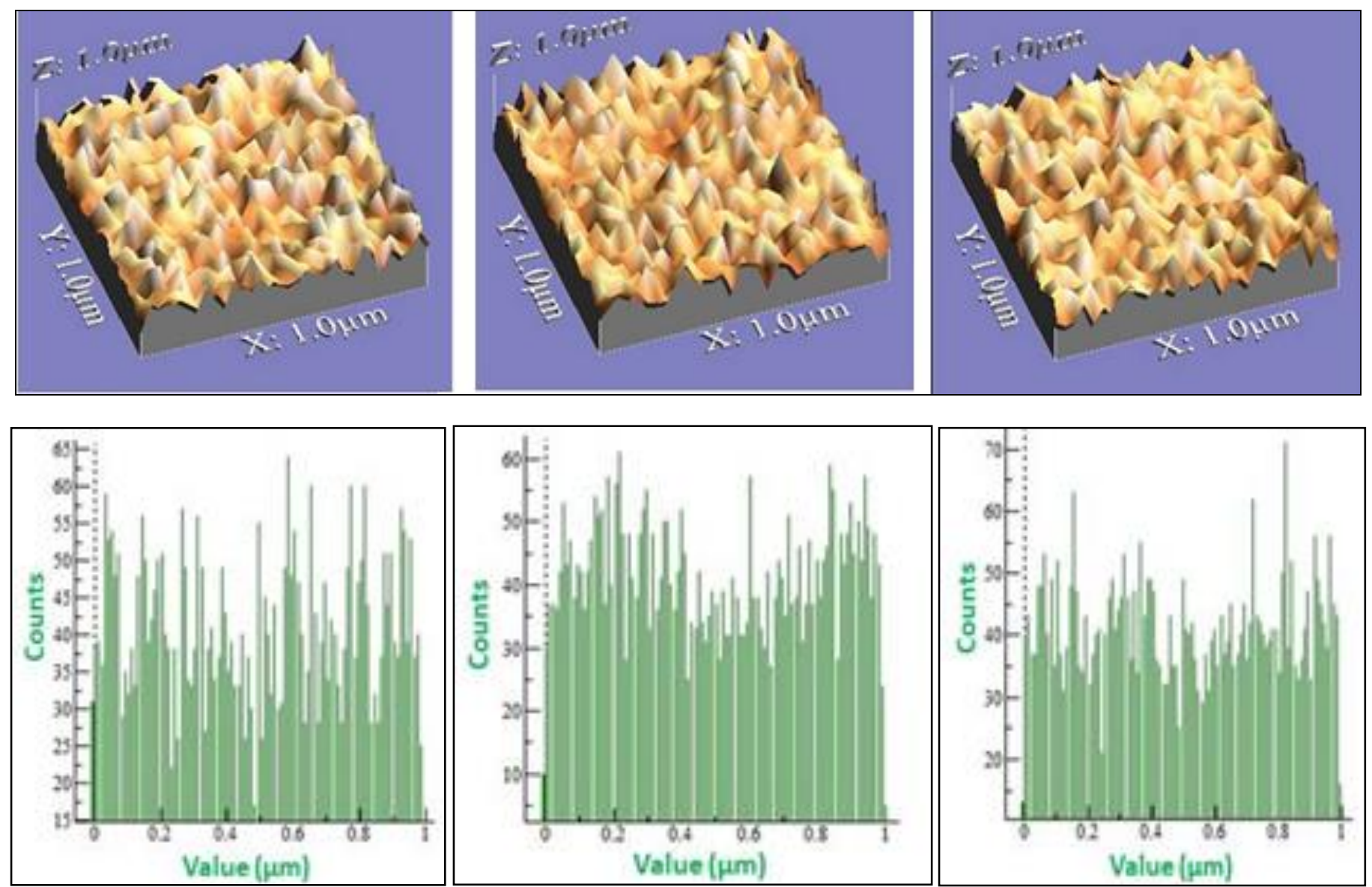

Fig 9: 3-D optical profilometry images and Histograms of polished zirconia reinforced lithium silicate (A1, B1), glazed zirconia reinforced lithium silicate (A2, B2) and glazed lithium disilicate (A3, B3) glass ceramics discs.

Results of surface roughness using Scanning Electron Microscope (SEM): According to the surface topographical features obtained from SEM, it was noticed that group (A) polished Celtra Duo® CAD reveled deep defects and well defined grooves Figure (10). Group $(B)$ Glazed Celtra Duo® CAD showed homogenous surface defects at $250 \mathrm{X}$ magnification. While when magnification increases, the surface became more homogenous and smoother Figure (11).On the other hand, group $(C)$ Glazed IPS e.max ${ }^{\circledR}$ CAD showed the most homogenous surface with least counts of defects, grooves and pores Figure (12). 


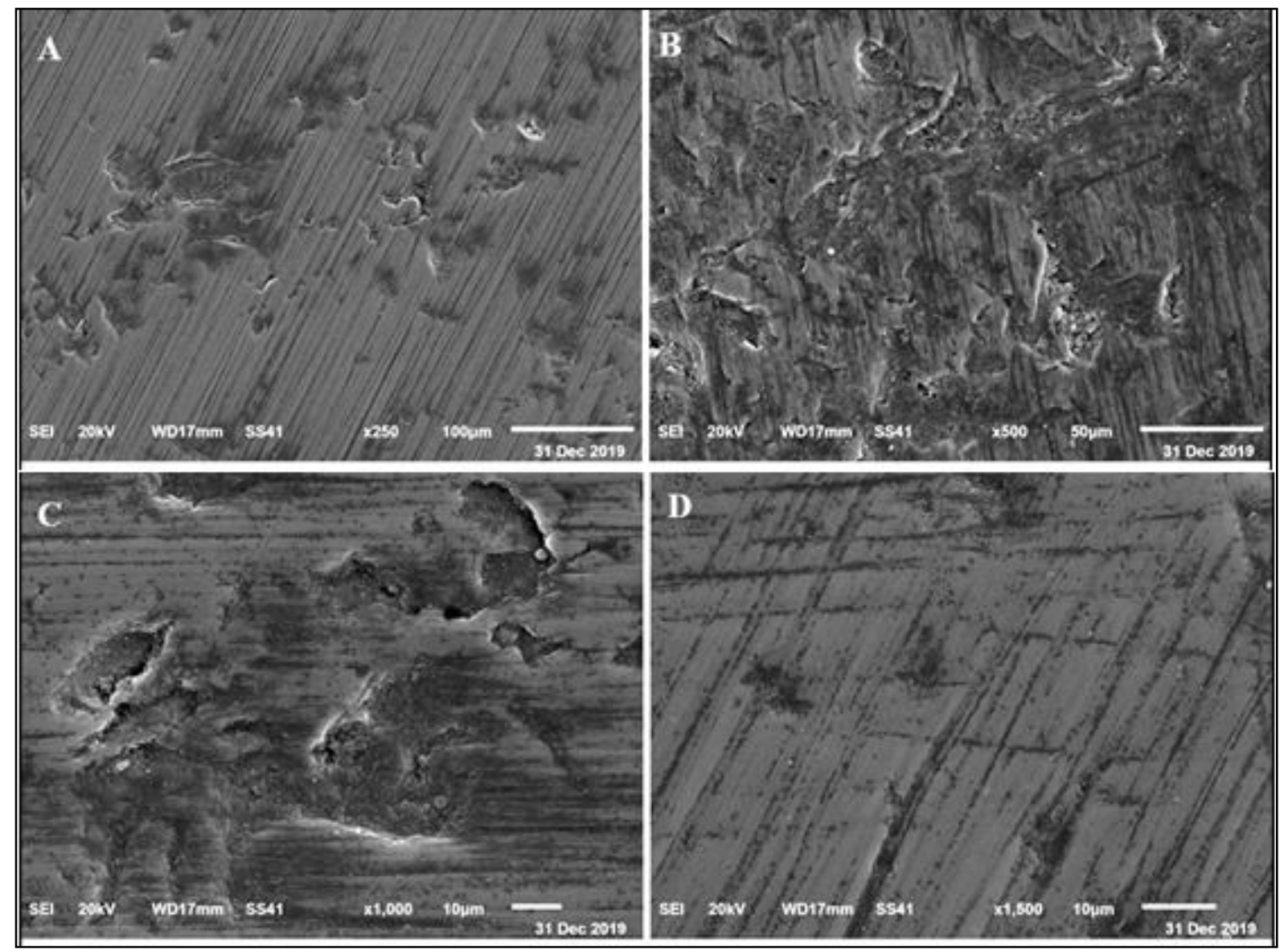

Fig 10: SEM micrograph of polished zirconia reinforced lithium silicate ceramic samples.

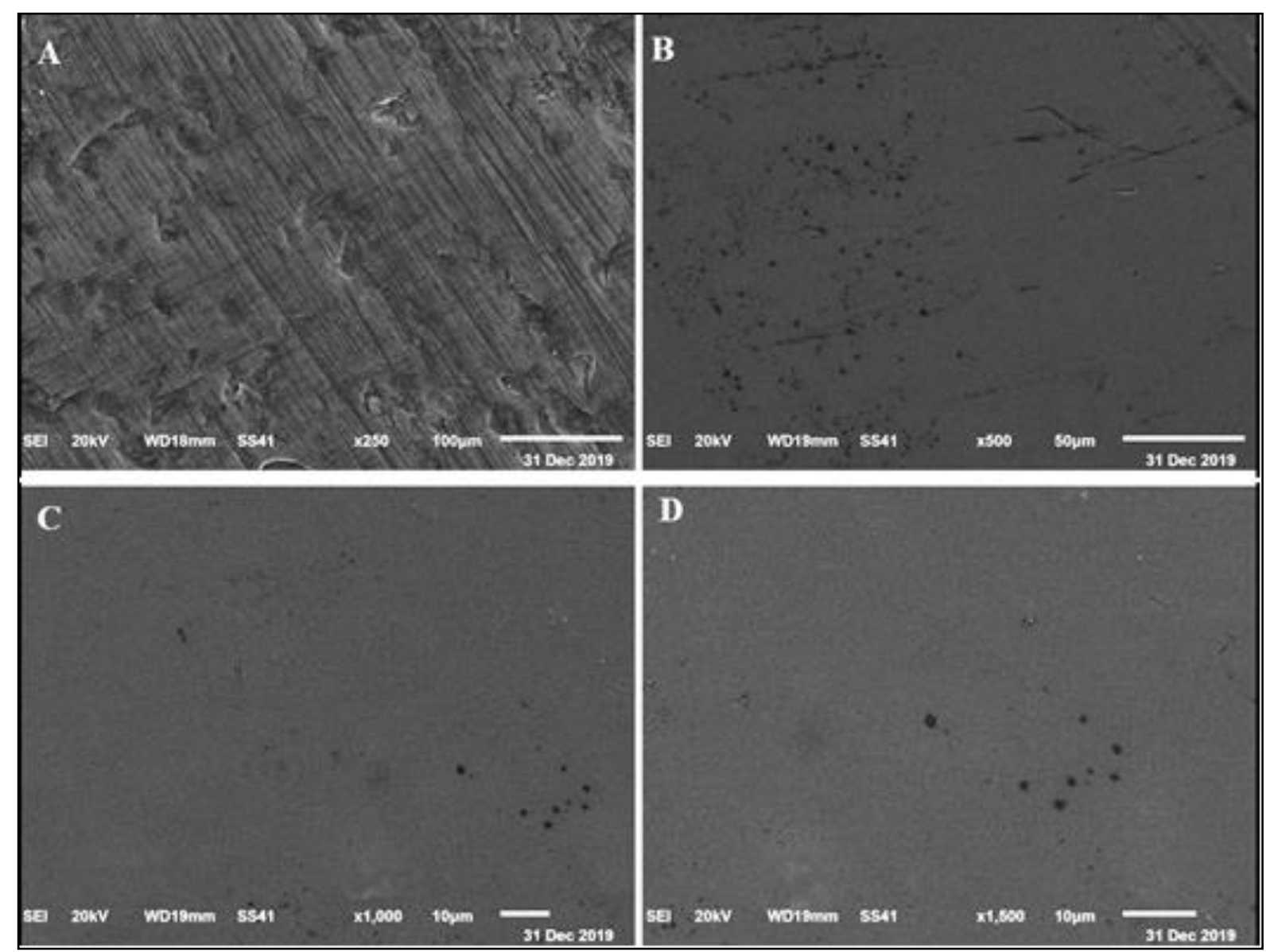

Fig 11: SEM micrograph of glazed zirconia reinforced lithium silicate ceramic samples 250X (A), 500X (B), $1000 \mathrm{X}$ (C) and 1500X (D). 

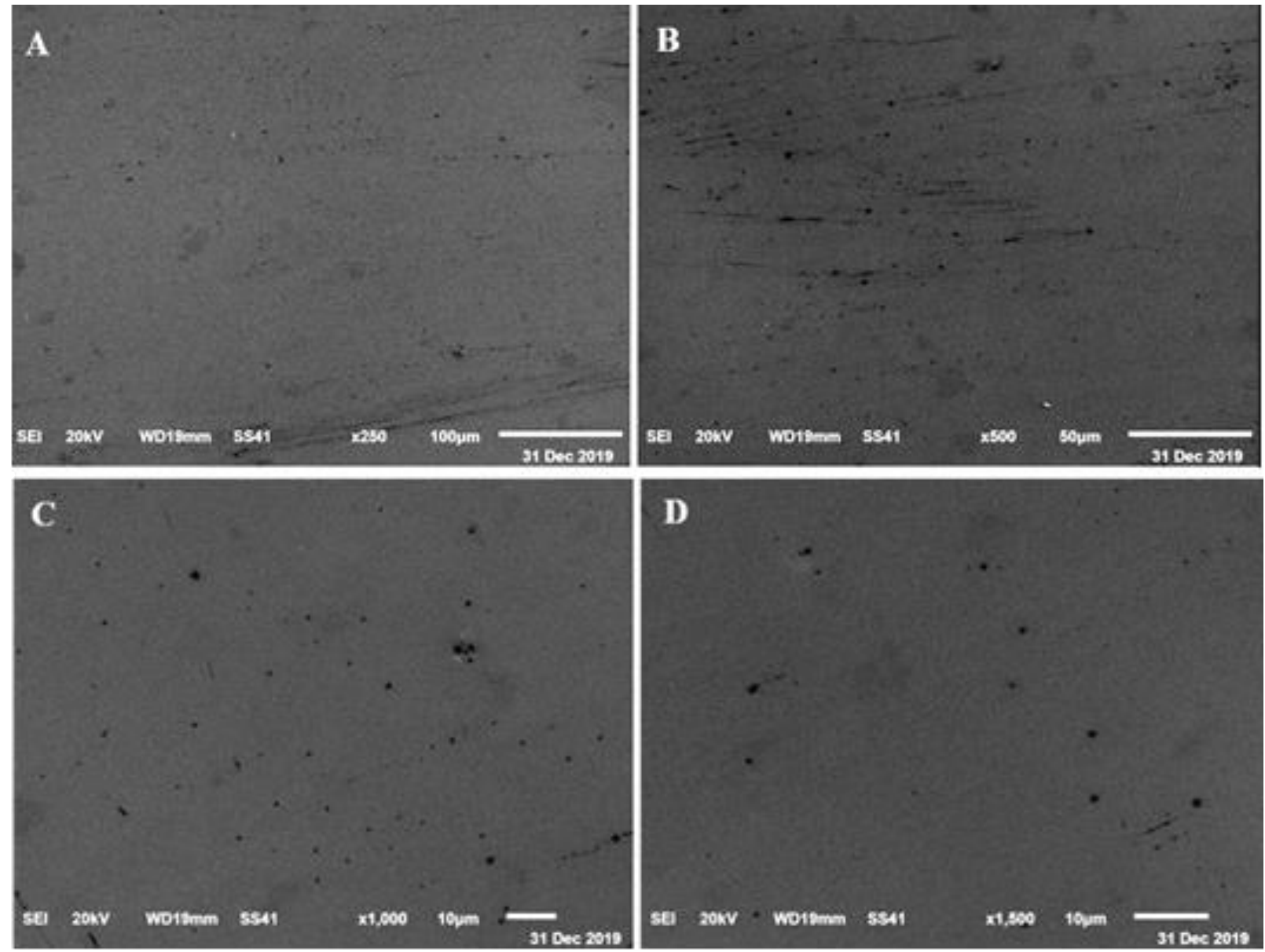

Fig 12: SEM micrograph of glazed lithium disilicate glass ceramic samples 250X (A), 500X (B), $1000 \mathrm{X}$ (C) and 1500X (D).

\section{Results of translucency testing}

Descriptive statistics showing mean values and standard deviations $( \pm \mathrm{SD})$ for translucency parameter $(\mathrm{TP})$ recorded for the 3 tested groups as affected by variables interaction (ceramic type and finishing protocol) are summarized in Table (2) and graphically represented in Figure (13).

It was found that Polished Celtra Duo recorded the highest translucency parameter mean values $(15.699 \mathrm{TP})$ followed by Glazed Celtra Duo mean values (13.915 TP) while the lowest translucency parameter mean values were recorded for Glazed IPS e.max (9.782 TP). The difference between groups was statistically significant as indicated by one-way ANOVA (p $=0.0062<0.05)$. Tukey's post-hoc test showed nonsignificant $(\mathrm{p}>0.05)$ difference between glazed and polished Celtra Duo.

\section{Results of translucency testing}

Descriptive statistics showing mean values and standard deviations $( \pm \mathrm{SD})$ for translucency parameter $(\mathrm{TP})$ recorded for the 3 tested groups as affected by variables interaction (ceramic type and finishing protocol) are summarized in Table (2) and graphically represented in Figure (13).

It was found that Polished Celtra Duo recorded the highest translucency parameter mean values (15.699 TP) followed by Glazed Celtra Duo mean values (13.915 TP) while the lowest translucency parameter mean values were recorded for Glazed IPS e.max (9.782 TP). The difference between groups was statistically significant as indicated by one-way ANOVA ( $\mathrm{p}$ $=0.0062<0.05)$. Tukey's post-hoc test showed nonsignificant $(\mathrm{p}>0.05)$ difference between glazed and polished Celtra Duo.

Table 2: Results of the translucency testing of the tested ceramics:

\begin{tabular}{|c|c|c|c|c|c|c|}
\hline \multirow{2}{*}{\multicolumn{2}{|c|}{ Variables }} & \multicolumn{2}{|c|}{ Descriptive Statistics } & \multicolumn{2}{|c|}{ 95\% Confidence Intervals } & \multirow{2}{*}{$\begin{array}{c}\text { ANOVA } \\
\text { P value } \\
\end{array}$} \\
\hline & & Mean & SD & Lower & Upper & \\
\hline \multirow{2}{*}{ Celtra Duo® CAD } & Polished (A) & $15.699 \mathrm{a}$ & 4.176 & 12.61 & 18.79 & \multirow{3}{*}{$0.0062 *$ sig } \\
\hline & Glazed (B) & $13.915 \mathrm{a}$ & 0.731 & 13.37 & 14.46 & \\
\hline IPS e.max ${ }^{\circledR}$ CAD & Glazed (C) & $9.782 \mathrm{~b}$ & 0.362 & 9.514 & 10.05 & \\
\hline
\end{tabular}

*Sign; significant $(P<0.05)$, different letters (a and $\mathrm{b})$ indicate statistically significant difference $(P<0.05)$ between groups. 


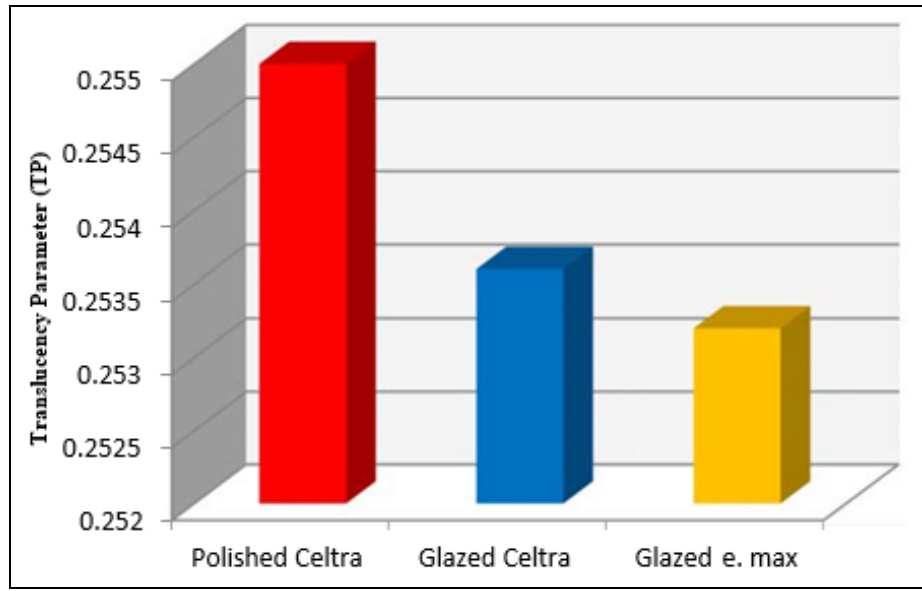

Fig 13: Column chart of the translucency testing of the tested ceramics.

\section{Discussion}

It is not yet known whether the zirconia reinforcement of glass ceramics (Celtra Duo) as well as finishing protocol (polishing vs. glazing) will affect its surface roughness and translucency compared to glass ceramics (Lithium Disilicate). The null-hypothesis of the present study was partially rejected, because there was significant effect of ceramic type on translucency. While there was no significant effect of finishing protocol on translucency. On the other hand, there was no-significant effect of neither the ceramic type nor the finishing protocol on surface roughness.

The zirconia particles incorporated where claimed to reinforce the ceramic structure by crack interruption, it has been assumed that the structure exhibits enhanced mechanical properties and fulfills the highest esthetic requirements at the same time. That's why zirconia reinforced lithium silicate was selected in the present study. In order to standardize shade and translucency, both materials were chosen in A2/HT shade, supposed to this shade were more commonly used in esthetic restorations of anterior teeth.

In the present study CAD/CAM technology was used to adhere to the uprising interest in using such technology, where this technique offered less time consumption, ease, patient's satisfaction and better reliability compared to other techniques ${ }^{[4]}$.

The results of surface finishing effect (polishing vs. glazing) on surface roughness of tested samples showed that the difference between groups was statistically non- significant as indicated by two-ANOVA test $(\mathrm{p}=0.6364>0.05)$ where (polished $\geq$ glazed). Thus, the null hypothesis that stated that the finishing protocol (polishing vs. glazing) of the zirconia reinforced lithium silicate (Celtra Duo® ${ }^{\circledR}$ CAD) will not affect the surface roughness was accepted.

This finding was in accordance with most of the studies that agree on the comparability of the two procedures regarding most ceramic materials Amer et al. (2015) ${ }^{[7]}$, Sarac et al. (2006) and Lawson et al. (2014) who reported less efficacy of glazing when compared with manual polishing ${ }^{[7,8,9]}$.

This finding was in disagreement with Aksoy et al. (2006) they concluded that, glazing decreased the surface roughness significantly irrespective of the prior treatment applied. This was also in disagreement with Maciel et al. (2019) who concluded that, polishing systems (Shofu Inc and Edenta AG) did not promote superficial smoothness similar to the glaze on the tested ceramics (IPS e.max Ceram e IPS e.max Press) $[10,11]$

The results of finishing protocol effect on translucency showed that the difference between groups was statistically non-significant between both tested finishing protocols as indicated by two-ANOVA test $(\mathrm{p}=0.0569>0.05)$ where (polished $\geq$ glazed). Thus, the null hypothesis speculated that the finishing protocol (polishing vs. glazing) of tested ceramics will not affect the translucency was accepted.

The difference in TP values might have originated from the different grain size and crystalline structure of the materials. The crystal size of zirconia-reinforced lithium silicate ceramic has been reported to be 4 to 8 times smaller than lithium disilicate ceramics after crystallization ${ }^{[12]}$.

The addition of $\mathrm{ZrO} 2$ increases the translucency of the glassceramics by varying the chemical and crystallization conditions, increase matrix viscosity and decrease crystal growth ${ }^{[13]}$. This finding was in agreement with Hayran and Sarıkaya (2019) who upon comparing different surface finishing methods on translucency of ZLS ceramic (Vita Suprinity) and lithium disilicate (IPS e.max CAD) found that the translucency parameter (TP) of polished Vita Suprinity was higher than glazed Vita Suprinity, while glazed IPS e.max has the lowest TP values. The difference in the TP values could be the result of the different dimensions and distribution of the ceramic material's crystals ${ }^{[14]}$.

On the other hand, this finding was in disagreement with Kurt et al. (2019) who found that, the TP values of the ZLS ceramic (Vita Suprinity) specimens were observed to be lower than lithium disilicate ceramic (IPS e.max) specimens in the all experimental groups (mechanical polishing before crystallization, mechanical polishing after- crystallization, glaze powder/liquid after crystallization, glaze paste before crystallization, glaze paste after crystallization, glaze spray before crystallization and glaze spray after crystallization) ${ }^{[15]}$. They attributed the difference in translucency to the difference in the crystalline content of the materials, where increasing the crystalline contents often result in greater opacity. Since no significant difference was found in the surface roughness with the tested variables. Then within the limitation of the present study; it could be stated that the translucency was affected by ceramic material type and not the surface roughness.

\section{References}

1. Bahgat SFA, Basheer RR, El Sayed SM. Effect of zirconia addition to lithium disilicate ceramic on translucency and bond strength using different adhesive strategies. Egypt Dent J 2015;61(4519):4533.

2. Raptis NV, Michalakis KX, Hirayama H. Optical behavior of current ceramic systems. Int J Periodontics Restorative Dent 2006;26(1):31-41. 
3. Monteiro JB, Riquieri H, Prochnow C, Guilardi LF, Pereira GKR, Borges ALS, et al. Fatigue failure load of two resin-bonded zirconia-reinforced lithium silicate glass-ceramics: Effect of ceramic thickness. Dent Mater J 2018;34(6):891-900.

4. Akar GC, Pekkan G, Çal E, Eskitaşçıoğlu G, Özcan M. Effects of surface-finishing protocols on the roughness, color change, and translucency of different ceramic systems. J Prosthet Dent 2014;112(2):314-321.

5. Kakaboura A, Fragouli M, Rahiotis C, Silikas N. Evaluation of surface characteristics of dental composites using profilometry, scanning electron, atomic force microscopy and gloss-meter. J Mater Sci Mater Med 2007;18(1):155-163.

6. Horcas I, Fernández R, Gomez-Rodriguez JM, Colchero JWSX, Gómez-Herrero JWSXM, Baro AM, et al. WSXM: a software for scanning probe microscopy and a tool for nanotechnology. Rev Sci Instrum 2007;78(1):013705.

7. Amer R, Kürklü D, Johnston W. Effect of simulated mastication on the surface roughness of three ceramic systems. J Prosthet Dent 2015;114(2):260-265.

8. Sarac D, Sarac YS, Yuzbasioglu E, Bal SA. The effects of porcelain polishing systems on the color and surface texture of feldspathic porcelain. $\mathrm{J}$ Prosthet Dent 2006;96(2):122-128.

9. Lawson NC, Janyavula S, Syklawer S, McLaren EA, Burgess JO. Wear of enamel opposing zirconia and lithium disilicate after adjustment, polishing and glazing. J Dent 2014;42(12):1586-1591.

Aksoy G, Polat H, Polat M, Coskun G. Effect of various treatment and glazing (coating) techniques on the roughness and wettability of ceramic dental restorative surfaces. Colloids Surf B Biointerfaces 2006;53(2):254259. 